\title{
von Willebrand factor, tissue plasminogen activator, and dehydroepiandrosterone sulphate predict cardiovascular death in a 10 year follow up of survivors of acute myocardial infarction
}

\author{
J-H Jansson, T K Nilsson, O Johnson
}

\begin{abstract}
Background-Haemostasis plays a major part in the process initiating a myocardial infarction. The impact of haemostatic variables on long term prognosis is unknown.

Objective-To evaluate von Willebrand factor (vWF), tissue plasminogen activator antigen (t-PA) and its activity before and after venous occlusion, plasminogen activator inhibitor (PAI-1), dehydroepiandrosterone sulphate (DHEAS), and established clinical risk factors as long term predictors for reinfarction and mortality. Patients-123 consecutive survivors of myocardial infarction followed up for 10 years.

Design-Study entry took place between 1982 and 1983. Fifty seven patients died (54 of cardiovascular disease) during the mean observation time of 10 years.

Results-Cox's univariate regression analysis showed that cardiovascular mortality was significantly associated with age, hypertension, previous history of angina pectoris, DHEAS, mass concentration of t-PA, and vWF. These associations were significant for $\mathrm{VWF}$ and mass concentration of t-PA after adjusting for age and hypertension.
\end{abstract}

Conclusions-A low concentration of DHEAS and high levels of the endothelially derived haemostatic variables VWF and mass concentration of t-PA are predictors of cardiovascular mortality in survivors of myocardial infarction. This association is independent of established clinical risk factors for mass concentration of t-PA and vWF.

(Heart 1998;80:334-337)

Internal Medicine, Skellefteå Hospital, Sweden

J-H Jansson

Departments of Clinical Chemistry and Internal Medicine, Umeå University Hospital, Sweden T K Nilsson

O Johnson

Correspondence to: Dr O Johnson, Department of Medicine, University Hospital of Umeå, S 90185 Umeå, Sweden.

Accepted for publication 23 March 1998
Keywords: myocardial infarction; risk factors; fibrinolysis; tissue plasminogen activator; plasminogen activator inhibitor; von Willebrand factor; dehydroepiandrosterone sulphate

Survivors of myocardial infarction have a hypofibrinolytic state, ${ }^{12}$ characterised by an increased mass concentration of tissue plasminogen activator ( $\mathrm{t}-\mathrm{PA}$ ) and increased activity of plasminogen activator inhibitor (PAI-1). Endothelially derived haemostatic factors, such as t-PA, PAI-1, and von Willebrand factor ( $\mathrm{vWF}$ ), predict cardiovascular complications in patients with vascular disorders, ${ }^{3-10}$ and even in apparently healthy men. ${ }^{11-13}$ It is reasonable to assume that these factors may have a role not only in thrombus formation but also in atherogenesis.

Dehydroepiandrosterone sulphate (DHEAS) is the adrenal androgen present in the highest concentration in the circulation. It may predict life expectancy and aging, ${ }^{14}$ but its precise physiological functions are uncertain. Beneficial effects on lipids ${ }^{15}$ and diabetes ${ }^{16}$ in animal models have been found, suggesting an antiatherogenic effect. An association has been reported between low plasma concentration of DHEAS and an increased risk of deep vein thrombosis after major abdominal surgery in humans. ${ }^{17}{ }^{18}$ Low levels of DHEAS have been found in young male survivors of myocardial infarction, ${ }^{19}{ }^{20}$ and in one study were independently and inversely related to cardiovascular mortality in men older than $50 .^{21}$

The cohort in most cited studies has been followed for a period of less than five years. The aim of the present study was to evaluate the long term prognosis of a patient group with a high cardiovascular mortality, with emphasis on the putative novel risk indicators DHEAS and the endothelially derived haemostatic factors.

\section{Patients and methods}

A total of 123 consecutive survivors of myocardial infarction (95 men and 28 women aged less than 70) were followed up at the outpatient clinic, department of internal medicine, Umeå University Hospital, between 20 November 1982 and 28 November $1983 .^{2}$ Blood sampling was performed three months after discharge from hospital. Patients who smoked at the time of myocardial infarction were considered as smokers and others as non-smokers. Hypertension was considered as present only in those patients who were clinically diagnosed as having this condition.

The study was approved by the local ethics committee. Informed consent was obtained from all participants. Table 1 lists the clinical and laboratory data at baseline.

\section{FOLLOW UP PROTOCOL}

Patients records were searched for death in November 1993. Death certificates were obtained. The cause of death was noted from the certificates which were of good validity. ${ }^{22}$ Cardiovascular death was defined according to the International Classification of Diseases, ninth 
Table 1 Clinical and laboratory variables at baseline

\begin{tabular}{|c|c|c|c|c|c|c|}
\hline & \multicolumn{3}{|c|}{ No cardiovascular death $(n=69)$} & \multicolumn{3}{|c|}{ Cardiovascular death $(n=54)$} \\
\hline & $n(\%)$ & Mean (SD) & $95 \% C I$ & $n(\%)$ & Mean (SD) & $95 \% C I$ \\
\hline Age (years) & 69 & $58(9)$ & 55 to 60 & 54 & $61(7)$ & 59 to 63 \\
\hline Male & $53(77)$ & & & $42(78)$ & & \\
\hline Female & $16(23)$ & & & $12(22)$ & & \\
\hline Hypertension & $16(23)$ & & & $21(39)$ & & \\
\hline Diabetes mellitus & 9 (13) & & & $6(11)$ & & \\
\hline Smoker & $25(36)$ & & & $11(20)$ & & \\
\hline Previous angina pectoris & $25(36)$ & & & $41(76)$ & & \\
\hline AST (units/1) & 63 & $4.3(3.1)$ & 3.5 to 5.1 & 53 & $4.2(3.3)$ & 3.3 to 5.1 \\
\hline Triglycerides $(\mathrm{mmol} / \mathrm{l})$ & 60 & $2.2(1.1)$ & 1.9 to 2.5 & 45 & $2.0(0.9)$ & 1.7 to 2.3 \\
\hline Cholesterol $(\mathrm{mmol} / \mathrm{l})$ & 61 & $7.1(1.5)$ & 6.7 to 7.5 & 46 & $6.9(1.2)$ & 6.5 to 7.3 \\
\hline DHEAS $(\mu \mathrm{mol} / \mathrm{l})$ & 69 & $6.0(3.9)$ & 5.0 to 6.9 & 54 & $4.6(2.7)$ & 3.8 to 5.3 \\
\hline SHBG $(\mu \mathrm{mol} / \mathrm{l})$ & 69 & $25(14)$ & 21 to 28 & 54 & $26(13)$ & 23 to 30 \\
\hline vWF (\% of normal) & 69 & $138(61)$ & 123 to 153 & 54 & $164(52)$ & 150 to 178 \\
\hline vWF vo (\% of normal) & 67 & $176(59)$ & 161 to 190 & 54 & $221(69)$ & 202 to 240 \\
\hline tPA activity $(\mathrm{U} / \mathrm{ml})$ & 69 & $0.09(0.07)$ & 0.07 to 0.11 & 54 & $0.12(0.13)$ & 0.08 to 0.16 \\
\hline tPA activity vo $(\mathrm{U} / \mathrm{ml})$ & 67 & $2.3(2.9)$ & 1.6 to 3.0 & 54 & $1.7(2.2)$ & 1.2 to 2.3 \\
\hline tPA mass conc $(\mu \mathrm{g} / 1)$ & 69 & $11.0(4.3)$ & 10.0 to 12.0 & 54 & $12.8(5.4)$ & 11.4 to 14.3 \\
\hline tPA mass conc vo $(\mu \mathrm{g} / \mathrm{l})$ & 67 & $35(23)$ & 29 to 40 & 54 & $40(28)$ & 33 to 48 \\
\hline PAI-1 (U/ml) & 69 & $10.6(7.1$ & 8.9 to 12.3 & 54 & $9.1(5.1)$ & 7.8 to 10.6 \\
\hline
\end{tabular}

AST, aspartate aminotransferase; DHEAS, dehydroepiandrosterone sulphate; SHBG, sexual hormone binding globulin; vWF von Willebrand factor; vo, after venous occlusion; tPA, tissue plasminogen activator; conc, concentration; PAI-1, plasminogen activator inhibitor.

edition (codes 390-459). Non-fatal infarction was not searched. All patients were followed up-that is, all records were found and evaluated.

BLOOD SAMPLING AND LABORATORY PROCEDURE Blood samples were taken when the acute phase of the disease had receded, three months after hospital discharge. Blood was obtained at rest and 10 minutes after venous occlusion (100 $\mathrm{mm} \mathrm{Hg}$ for 10 minutes) for preparation of citrated plasma and assay for t-PA to evaluate mass concentration and activity. PAI-1 activity and vWF were measured from samples taken only at rest. t-PA and PAI-1 activities were measured by a chromogenic substrate assay, while mass concentration of t-PA and vWF were measured with an enzyme linked immunosorbent assay. Further details have been described by Nilsson and Johnson. ${ }^{2}$

Plasma DHEAS was measured directly in diluted plasma with a radioimmunoassay, using an antiserum (Endocrine Sciences, California, USA) raised against DHEA-SO $\mathrm{SO}_{4}$-17-oxime-

Table 2 Relation between risk indicators and cardiovascular mortality (Cox regression analysis)

\begin{tabular}{lcccc}
\hline & \multicolumn{2}{c}{ Univariate } & & \multirow{2}{*}{ Multivariate } \\
\cline { 2 - 3 } & $p$ value & Risk ratio $(95 \%$ CI $)$ & & \\
\hline Age & 0.034 & $1.045(1.003$ to 1.088$)$ & \\
Sex & 0.40 & & \\
Hypertension & 0.033 & $1.817(1.050$ to 3.143$)$ & \\
Diabetes mellitus & 0.40 & & \\
Smoker & 0.18 & & \\
Previous angina pectoris & $<0.001$ & $3.982(2.129$ to 7.499$)$ & \\
AST & 0.40 & & \\
Triglycerides & $>0.40$ & & \\
Cholesterol & $>0.40$ & & \\
DHEAS & 0.029 & $0.892(0.804$ to 0.989$)$ & \\
SHBG & 0.40 & & \\
vWF & 0.009 & $1.005(1.001$ to 1.008$)$ & \\
vWF vo & $<0.001$ & $1.008(1.004$ to 1.012$)$ & \\
tPA activity & 0.070 & & $0.025 \dagger$ \\
tPA activity vo & 0.27 & & \\
tPA mass conc & 0.023 & $1.063(1.009$ to 1.121$)$ & \\
tPA mass conc vo & 0.17 & & \\
PAI-1 & 0.34 & & \\
\hline
\end{tabular}

${ }^{\star}$ Only significant values are shown; †After exclusion of vWF and DHEAS.

AST, aspartate aminotransferase; DHEAS, dehydroepiandrosterone sulphate; SHBG, sexual hormone binding globulin; vWF von Willebrand factor; vo, after venous occlusion; tPA, tissue plasminogen activator; conc, concentration; PAI-1, plasminogen activator inhibitor. bovine serum albumin; $7-{ }^{3} \mathrm{H}-\mathrm{DHEAS}$ was used as tracer and free and bound radioactivity were separated by precipitation using ammonium sulphate.

\section{STATISTICAL METHODS}

Baseline variables were divided into quartiles $\left(Q_{1}-Q_{4}\right)$ and the incidence of mortality in each quartile was calculated per 100 patient-years to show the relation between a possible prognostic factor and the incidence of cardiovascular mortality. These quartiles were not used to test relations. Relative risk was calculated as the incidence of mortality in $\mathrm{Q}_{4}$ per incidence of mortality in $\mathrm{Q}_{1}$.

Cox's regression analysis ${ }^{23}$ was performed to test for a relation between a baseline variable and the incidence of mortality. Variables with a significant relation in a univariate analysis were studied further in a backward stepwise multivariate analysis. Relations for continuous variables were considered as linear. Two tailed tests were performed and $\mathrm{p}<0.05$ was considered significant.

\section{Results}

During the 10.5 (0.3) year follow up 57 patients $(46 \%)$ died: 54 of cardiovascular disease (51 of coronary heart disease, one of stroke, and two of pulmonary embolism), one of pulmonary cancer, one of respiratory insufficiency in the terminal state of amyotrophic lateral sclerosis, and one of a methadone overdose. Mortality was similar in men and women (table 1). There was no difference in the use of diuretics three months after myocardial infarction between patients who subsequently died of cardiovascular disease and others, indicating similar numbers of patients with clinical signs of heart failure in these groups.

Cox's univariate analyses with cardiovascular mortality as the response variable showed a significant negative association with DHEAS and significant positive associations with age, hypertension, previous angina pectoris, mass concentration of t-PA, and vWF (table 2). DHEAS was significantly and negatively corre- 


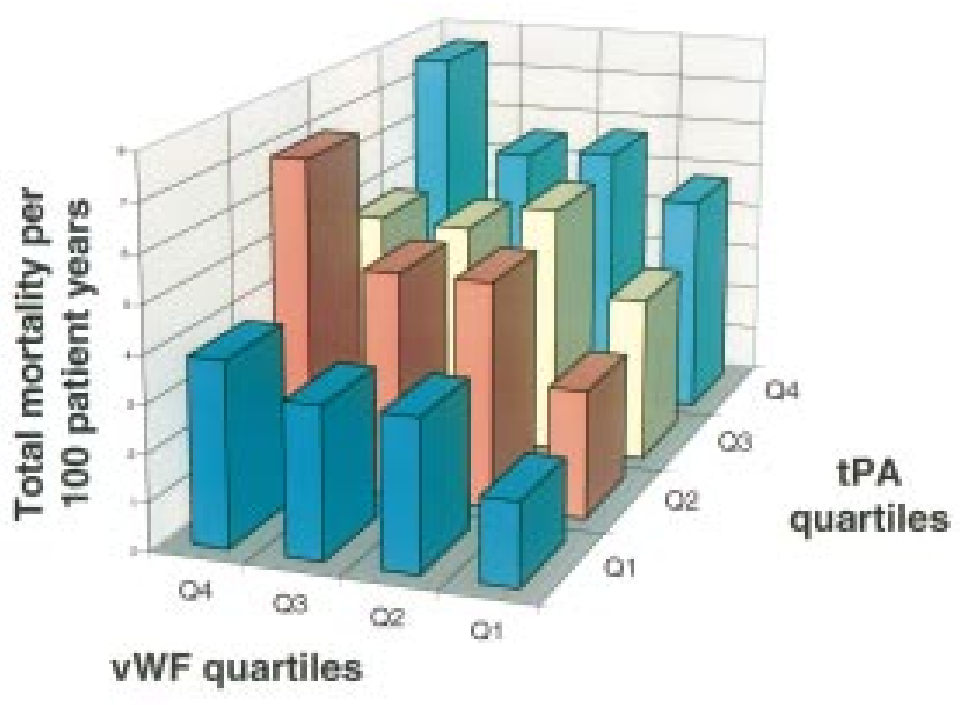

Figure 1 Relation between the incidence of cardiovascular mortality (per 100 patient-years) after 10 years'follow up and quartiles $Q 1$ to $Q 4$ of the mass concentration of tissue plasminogen activator $(t-P A)$ and von Willebrand factor $(v W F)$.

lated to age $(r=-0.48)$. Thus, less than $25 \%$ variation in DHEAS can be explained by age. Relative risk (incidence of mortality in $\mathrm{Q}_{4}$ per incidence of mortality in $\mathrm{Q}_{1}$ ) for 10 year cardiovascular mortality was 2.2 (95\% confidence interval (CI) 0.98 to 4.72 ) with t-PA as the marker, 2.6 (95\% CI 1.09 to 6.27) with vWF, and 0.56 (95\% CI 0.27 to 1.19$)$ with DHEAS.

Cox's multivariate regression analyses showed that $\mathrm{vWF}(\mathrm{p}=0.0061, r=0.0052)$ and pharmacological treatment for hypertension ( $p=0.0229, r=0.6411$ ) were independently associated with cardiovascular mortality at 10 year follow up when clinical and laboratory risk factors were entered as independent variables. Continuous variables were age, triglycerides, cholesterol, aspartate aminotransferase, vWF, and PAI-1 activity; discrete variables were sex, smoking habits (nonsmoker, current smoker), pharmacological treatment of hypertension, and diabetes mellitus. Mass concentration of t-PA was independently associated with cardiovascular mortality ( $p=0.025, r=0.064$ ) when the same analyses were performed after exclusion of vWF and DHEAS. The relation between DHEAS and cardiovascular mortality remained after adjustment for hypertension ( $p=0.0495)$ but not after adjustment for age $(\mathrm{p}=0.155)$.

Figure 1 shows that the mass concentration of t-PA and vWF have a mutual independent association with mortality.

Correlations between vWF and the mass concentration of t-PA were 0.299 $(\mathrm{p}=0.0008)$, between $\mathrm{vWF}$ and DHEAS $-0.164(\mathrm{p}=0.070)$, and between t-PA and DHEAS $-0.068(\mathrm{p}=0.45)$.

\section{Discussion}

Survivors of myocardial infarction have a high risk of cardiovascular death. Our results suggest that long term risk may be predicted by DHEAS, vWF, and the mass concentration of t-PA. The recently published European concerted action on thrombosis and disabilities angina pectoris study on a large cohort of patients with angina pectoris also showed a strong association between cardiovascular events during a two year follow up and vWF and mass concentration of t-PA ${ }^{9}$ : both factors were interpreted as representing endothelial dysfunction. In fig 1, however, these two factors independently predict the risk of mortality and thus they are unlikely to represent merely a single common mechanism.

In our study low concentrations of DHEAS were associated with cardiovascular mortality. Nevertheless, after adjustment for age there was no significant relation between DHEAS and cardiovascular mortality.

A decreased concentration of DHEAS is found in men with coronary artery disease compared with that measured in controls and there seems to be a relation between DHEAS levels and the extent of coronary atherosclerosis. ${ }^{24}$ Furthermore, some prospective studies show an inverse association between DHEAS and cardiovascular events, ${ }^{21}{ }^{25} 26$ however in one study this association disappeared after adjusting for the effects of age, ${ }^{24}$ as seen in the present study.

vWF is essential in the aggregation of platelets and the formation of thrombi, particularly in stenotic arteries with high shear stress. ${ }^{27}$ Monoclonal antibody against $\mathrm{VWF}$ in an extracorporeal pig perfusion system produced an $80 \%$ reduction in platelet deposition after superficial and deep vascular injury compared with a $30 \%$ reduction in swine treated with aspirin alone. The experimental evidence of vWF in thrombosis, which was recently reviewed by Bowie, ${ }^{28}$ provides a theoretical framework that fits well with the clinical results presented here and in previous studies. ${ }^{569}$

In our study the $95 \%$ CI for the relative risks (incidence of mortality in $\mathrm{Q}_{4}$ per incidence of mortality in $\mathrm{Q}_{1}$ ) of t-PA and vWF are wide, probably because of the small sample size. Nevertheless, Cox's regression analysis showed an independent statistically significant association between these variables and cardiovascular mortality. Clearly, our results that t-PA and VWF are better predictors for a poor long term prognosis than established risk factors will have to be confirmed in larger prospective studies. Whether these haemostatic variables are predictors even in individuals without established coronary heart disease is another important issue for future investigation.

Patients with coronary heart disease have increased PAI-1 activity compared with that in controls. ${ }^{12459}$ PAI-1 activity in young men with acute myocardial infarction before the age of 45 was associated with recurrent infarction in one study, ${ }^{3}$ but not in others on middle aged or older patients. ${ }^{459}$ Mass concentration of PAI-1 ("PAI-1 antigen"), which is strongly correlated with PAI-1 activity, was found to be related to cardiovascular events in univariate analysis but after adjustment for other risk factors this association disappeared. ${ }^{9}{ }^{10}$ Our finding that PAI- 1 activity did not predict long term events fits well with findings in previous short term studies on comparable patient populations. 
Several established risk factors, such as sex, smoking habits, diabetes mellitus, cholesterol, and triglycerides, were not significantly associated with cardiovascular mortality. Likewise, in the much larger ECAT study serum cholesterol was not associated with cardiovascular events. ${ }^{9}$ In the Scandinavian simvastatin survival study the placebo group included 2222 patients with verified ischaemic heart disease, $79 \%$ with previous myocardial infarction. ${ }^{29}$ On follow up there was a slight increasing incidence of major coronary events in the higher quartiles of serum cholesterol (relative risk $\left(\mathrm{Q}_{4} / \mathrm{Q}_{1}\right)$ 1.18). These negative findings do not imply that such factors are unimportant, but reflect that our study was not designed to evaluate these traditional risk factors. However, relations between DHEAS or the haemostatic factors and cardiovascular mortality are significant, indicating that they offer a stronger prediction of long term risk than established risk factors.

In conclusion, the endothelially derived haemostatic variables vWF and mass concentration of t-PA but not the steroid DHEAS were independently associated with mortality, indicating that they provide some independent predictive value for cardiovascular mortality in survivors of myocardial infarction.

Expert statistical advice was given by Kjell Pennert, Gothenburg. This study was supported by grants from the Swedish Medical Research Council (grant no 08267) and the Swedish Heart and Lung Fund.

1 Hamsten A, Wiman B, De Faire U, et al. Increased plasma levels of a rapid inhibitor of tissue plasminogen activator in
young survivors of myocardial infarction. $N$ Engl $7 \mathrm{Med}$ young survivors of

2 Nilsson TK, Johnson O. The extrinsic fibrinolytic system in survivors of myocardial infarction. Thromb Res 1987;48 621-30.

3 Hamsten A, DeFaire U, Walldius G, et al. Plasminogen activator inhibitor in plasma: risk factor for recurrent myocardial infarction. Lancet 1987;ii:3-9.

4 Jansson JH, Nilsson TK, Olofsson BO. Tissue plasminogen activator and other risk factors as predictors of cardiovascular events in patients with severe angina pectoris. Eur Heart f 1991;12:157-6

5 Jansson J H, Nilsson TK, Johnson O. von Willebrand factor in plasma: a novel risk factor for recurrent myocardial infarction and death. Br Heart f 1991;66:351-5.

6 Cortellaro M, Boschetti C, Cofrancesco E, et al. The PLAT study: hemostatic function in relation to atherothrombotic ischemic events in vascular disease patients. Principal ischemic events in vascular disease patients. Principal
results. Arteriosclerosis and Thrombosis 1992;12:1063-70.

7 Korninger C, Lechner K, Niessner H, et al. Impaired fibriKorninger C, Lechner K, Niessner $\mathrm{H}$, et al. Impaired fibri-
nolytic capacity predisposes for recurrence of venous nolytic capacity predisposes for recurrence
thrombosis. Thromb Haemost 1984:52:127-30.

8 Haines AP, Howarth D, North WRS, et al. Haemostatic variables and the outcome of myocardial infarction. Thromb Haemost 1983;50:800-3.
9 Thompson GS, Kienast J, Pyke S, et al. Hemostatic factors and the risk of myocardial infarction or sudden death in patients with angina pectoris. N Engl F Med 1995;332:63541 .

10 Brännström $\mathrm{M}$, Jansson JH, Boman $\mathrm{K}$, et al. Endothelial haemostatic factors may be associated with mortality in patients on long-term anticoagulant treatment. Thromb Haemost 1995;74:612-15.

11 Meade TW, Mellows S, Brozovic M, et al. Haemostatic function and ischemic heart disease: principal results of the Northwick Park heart study. Lancet 1986;ii:533-7.

12 Ridker PM, Vaughan DE, Stampfer MJ, et al. Endogenous tissue-type plasminogen activator and risk of myocardial infarction. Lancet 1993;341:1165-8.

13 Meade TW, Ruddock V, Stirling Y, et al. Fibrinolytic activity, clotting factors, and long-term incidence of ischaemic heart disease in the Northwick Park heart study. Lancet 1993;342:1076-79.

14 Lopez-S, A. Metabolic and endocrine factors in ageing. In: Rothschild H, Chapman, CF, eds. Risk factors for senility. New York: Oxford University Press, 1984:205.

15 Ben-David M, Dikstein S, Bismuth G, et al. Antihyperchoesterolemic effect of dehydroepiandrosterone in rats. Proc Soc Exp Biol Med 1967;125:1136.

16 Coleman DL, Leiter EH, Schwitzer RW. Therapeutic effects of dehydroepiandrosterone (DHEA) in diabetic mice. Diabetes 1982;31:830.

17 Nilsson T, Mellbring G, Damber JE. Relationships between tissue plasminogen activator, steroid hormones and deep vein thrombosis. Acta Chir Scand 1985;151:515-19.

18 Mellbring G, Chotai J, Nilsson TK. Preoperative plasma dehydroepiandrosterone sulphate concentrations in relation to postoperative deep vein thrombosis after major abdominal surgery. Surgical Research Communications 1989; 6:29-34.

19 Mitchell LE, Sprecher DL, Borecki IB, et al. Evidence for an association between dehydroepiandrosterone sulphate and nonfatal, premature myocardial infarction in males. Circulation 1994;89:89-93.

20 Slowinska-Srzednicka J, Zgliczynski S, CiswickaSznajderman, et al. Decreased plasma dehydroepiandrosterone sulphate and dihydrotestosterone concentrations in young men after myocardial infarction. Atherosclerosis young men after

21 Barret-Connor E, Khaw KT, Yen SSC. A prospective study of dehydroepiandrostenedione sulfate, mortality, and cardiovascular disease. N Engl F Med 1986;315:1519-24.

22 de Faire U, Friberg L, Lorich U, et al. A validation of causeof-death certification in 1156 deaths. Acta Med Scand 1976;200:223-8.

23 Cox DR. Regression models and life tables [with discussion]. F R Stat Soc 1972;34:187-220.

24 Herrington DM, Gordon GB, Achuff SC, et al. Plasma dehydroepiandrosterone and dehydroepiandrosterone sulphate in patients undergoing diagnostic coronary angiography. $f$ Am Coll Cardiol 1990;16:862-70.

25 Contoreggi CS, Blackman MR, Andres R, et al. Plasma levels of estradiol, testosterone and DHEAS do not predict risk of coronary artery disease in men. $f$ Androl 1990;11:460-70.

26 LaCroix AZ, Yano K, Reed D. Dehydroepiandrosterone sulfate, incidence of myocardial infarction, and extent of atherosclerosis in men. Circulation 1992;86:1529-32.

27 Gralnick HR, Williams SB, Mc Keown LP, et al. Platelet von Willebrand factor. Mayo Clinics Proceedings 1991;66:63440 .

28 Bowie EJW. The role of von Willebrand factor in thrombosis. In: Poller L, Tompson JM, eds. Thrombosis and its mansis. In: Poller L, Tompson JM, eds. Thrombosis and its man-

29 Scandinavian Simvastatin Survival Group. Baseline serum cholesterol and treatment effect in the Scandinavian simvastatin survival study (4S). Lancet 1995;345:1274-5. 